\title{
Antiterrordatei-Urteil: Fäusteschütteln in Richtung Luxemburg
}

VB verfassungsblog.de/antiterrordatei-urteil-fausteschutteln-in-richtung-luxemburg/

Maximilian Steinbeis Mi 24 Apr 2013

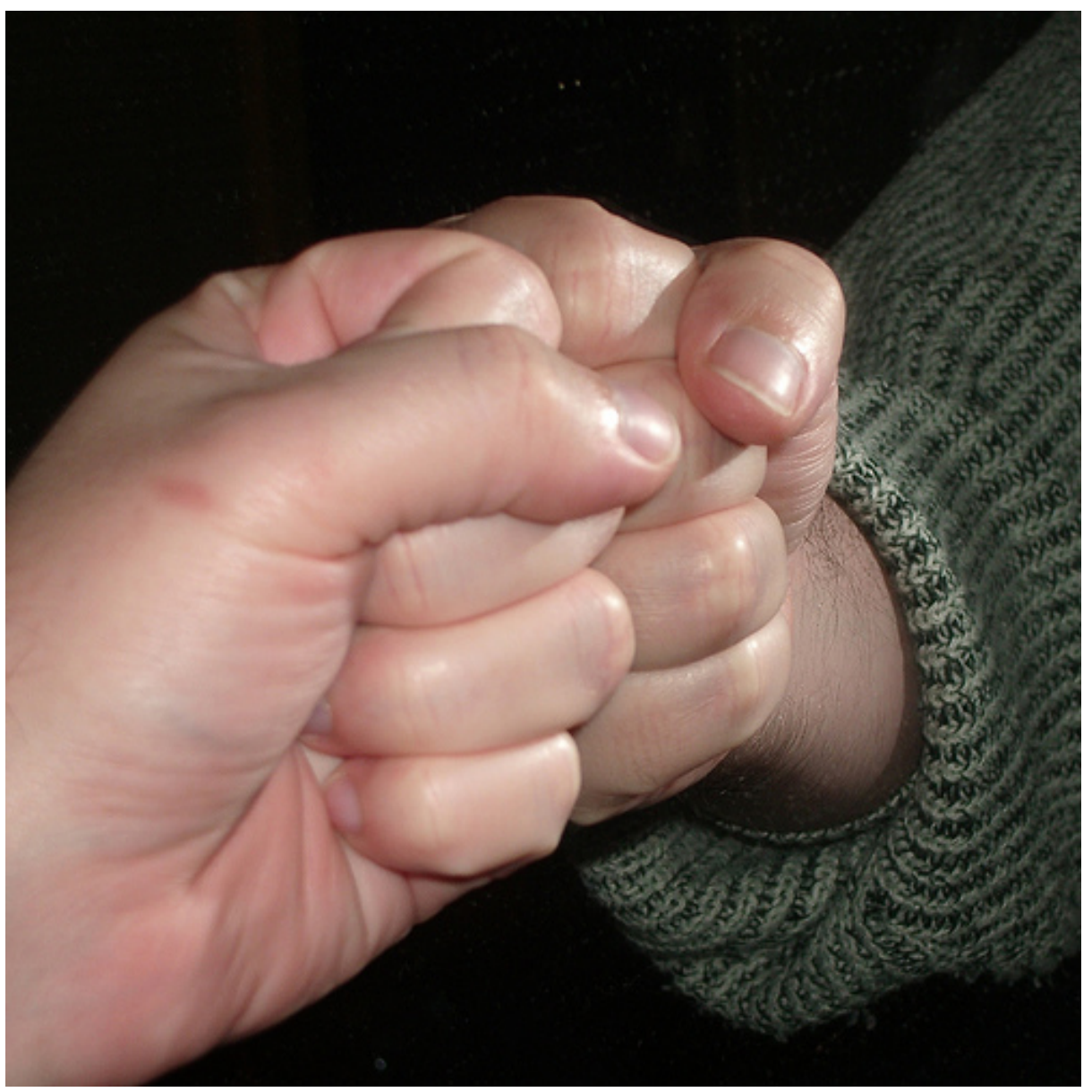

(c) GLindsay65, Flickr CC BY-ND 2.0

Die Antiterrordatei ist im Prinzip verfassungsmäßig, im Detail jedoch nicht, und dazu gäbe es eine Menge zu sagen. Zu Datenschutz und Trennungsgebot wird man heute noch viel lesen können. Ich will mich hier erst einmal auf einen Punkt konzentrieren, der mit dem eigentlichen Verfahrensgegenstand nur auf sehr verschlungenen Wegen zu tun hat: die ziemlich unverhohlene Aufforderung an den EuGH, seine Grundrechtsrechtsprechung zurückzustecken. Eine Aufforderung, der der Senat mit der ebenso unverhohlenen Drohung Nachdruck verleiht, sonst die Ultra-ViresBazooka herauszuholen. Der Erste Senat, wohlgemerkt. Und zwar einstimmig.

Um diese Art freundschaftlichen Meinungsaustauschs im europäischen Verfassungsgerichtsverbund pflegen zu können, schiebt der Senat eigens zwischen Zulässigkeit und Begründetheit einen speziellen Gliederungspunkt ein: Darin legt er dar, warum er in diesem Fall nicht verpflichtet ist, den EuGH per Vorlageverfahren um europarechtliche Klärung zu bitten. Der Fall habe nichts mit Europarecht zu tun, insbesondere nicht mit dem europäischen Grundrechtsschutz. Dass Antiterrordatei-Daten in Europa weitergegeben werden müssen, mache die Antiterrordatei nicht zu einem Anwendungsfall für die europäische Grundrechtecharta. Es sei nicht zu bezweifeln, dass der EuGH oder irgendein anderes Gericht in der EU das anders sehen (Acte-Claire-Rechtsprechung des EuGH). 
Wenn das alles nicht ist, wozu dann der ganze Aufwand? Die Antwort liegt auf der Hand: Åkerberg Fransson.

So heißt ein vor wenigen Wochen ergangenes Urteil des EuGH, das besagt, dass alle Fälle, die im Anwendungsbereich des Unionsrechts liegen, am Maßstab der europäischen Grundrechtecharta zu messen und somit im Zweifel dem EuGH vorzulegen sind - auch wenn der Anwendungsbereich nur am Rande berührt ist. Das ist er bei der europarechtlichen Durchdringung fast aller Rechtsbereiche in den allermeisten Fällen. Was wiederum bedeuten würde, dass Grundrechtsfragen künftig im Regelfall von Luxemburg beantwortet würden und nicht mehr von Karlsruhe.

Dem will Karlsruhe aber nicht tatenlos zusehen: Åkerberg Fransson, so der Senat, dürfe nicht so verstanden werden, dass

jeder sachliche Bezug einer Regelung zum bloß abstrakten Anwendungsbereich des Unionsrecht oder rein tatsächliche Auswirkungen auf dieses

genüge, um die Regelung voll der Grundrechtecharta zu unterwerfen. Denn anderenfalls wäre die ÅkerbergFransson-Entscheidung

offensichtlich als Ultra-vires-Akt zu beurteilen (...) oder Schutz und Durchsetzung der mitgliedstaatlichen Grundrechte in einer Weise (gefährdet), dass dies die Identität der durch das Grundgesetz errichteten Verfassungsordnung in Frage stellte.

Eine solche Lesart sei

im Sinne eines kooperativen Miteinanders zwischen dem Bundesverfassungsgericht und dem Europäischen Gerichtshof

zu vermeiden.

Eine Frage, die ich mir da stelle: Wenn hier so viel von möglichen Lesarten und Deutungsmöglichkeiten des Åkerberg-Fransson-Urteils die Rede ist - ja, warum hat denn dann der Senat nicht vorgelegt? Wie verträgt sich diese Passage mit der wenige Zeilen zuvor geäußerten Meinung, es handle sich um einen unzweifelhaften Fall im Sinne der Acte-Claire-Rechtsprechung? Zumal erst vor eineinhalb Jahren der selbe Erste Senat in der selben Besetzung im Fall Investitionszulagengesetz in klaren Worten beschrieben hat, wie hoch die Hürde für Acte Claire ist:

\footnotetext{
Ein letztinstanzliches nationales Gericht darf einen vernünftigen Zweifel an der Entscheidung der gestellten Frage nur verneinen, wenn es überzeugt ist, dass auch für die Gerichte der übrigen Mitgliedstaaten und den Europäischen Gerichtshof die gleiche Gewissheit bestünde. Nur wenn diese Voraussetzungen erfüllt sind, darf das letztinstanzliche innerstaatliche Gericht davon absehen, diese Frage dem Europäischen Gerichtshof vorzulegen und sie stattdessen in eigener Verantwortung beantworten
}

Dass für den Europäischen Gerichtshof die gleiche Gewissheit bestünde... Hm. Weiß nicht. Scheint mir jetzt nicht so 
total unbezweifelbar, ehrlich gesagt.

Tatsächlich macht das nur Sinn, wenn man die Sache durch die Ultra-Vires-Brille betrachtet, die sich das BVerfG mit dem Maastricht-Urteil aufgesetzt hat: Es handelt sich hier um einen Fall, in dem zwar mehrere Lesarten möglich sind, aber manche sind nach Meinung des BVerfG ultra vires, andere nicht. Die angeblichen ultra-vires-Lesarten stehen dem EuGH gar nicht zu Gebote, sie werden von Karlsruhe schon vorher abgefangen, bevor sie überhaupt über die Integrationsbrücke in den Wahrnehmungsbereich des EuGH gelangen. Nur die enge intra-vires-Lesart kann das Karlsruher Brückenwärterhäuschen passieren, und so ist das, was drüben am europäischen Ufer ankommt, tatsächlich ein wunderbar eindeutiger Acte Claire.

Der Erste Senat hat damit wieder einmal klar gemacht, wer seiner Meinung nach der Ober und wer der Unter ist in punkto Kompetenzbereich der EU.

Wenn es nur Karlsruhe und Luxemburg gäbe, wäre mir das ja Recht: Das Gleichgewicht des Schreckens zwischen diesen beiden Gerichten ist seit einem halben Jahrhundert stabil, und bisher hat sich noch jedes Eskalationsszenario in eine Notfall- und Reservekompetenz nach dem "Solange"-Muster auflösen lassen.

Dieses idyllische Brücken- und Gleichgewichtsbild, das wir da zu zeichnen gewohnt sind, trügt aber: Das ist keine symmetrische Konstellation, kein Tauziehen, in dem Karlsruhe am einen Ende zieht und Luxemburg am anderen. Es gibt nicht nur Karlsruhe und Luxemburg. Es gibt auch andere EU-Staaten, in denen die Frage, ob der EuGH die Grundrechte von Unionsbürgern auch gegen ihren eigenen Staat schützen darf, ganz handfest und konkret auf dem Tisch liegt. Was aus den Ungarn wird, kann Karlsruhe egal sein - Luxemburg aber nicht (oder Straßburg, for that matter). Was das für den europäischen Verfassungsgerichtsverbund bedeutet, diese Frage haben wir gerade erst begonnen uns zu stellen.

\section{LICENSED UNDER CC BY NC ND}

SUGGESTED CITATION Steinbeis, Maximilian: Antiterrordatei-Urteil: Fäusteschütteln in Richtung Luxemburg, VerfBlog, 2013/4/24, http://verfassungsblog.de/antiterrordatei-urteil-fausteschutteln-in-richtung-luxemburg/. 